\title{
El mito de la belleza y los cuerpos negros
}

The Myth of Beauty and Black Bodies

\section{DIANA KATHERINE MORENO GÓMEZ}

Psicóloga por la Universidad del Valle, Colombia, y magíster en Psicología Positiva por la Universidad de Jaén, España, actualmente se encuentra vinculada al Centro de Estudios Afrodiaspóricos (CEAF) de la Universidad Icesi, en Cali, Colombia.

\section{ISIS YAEL AMADOR CAMPUSANO}

Estudiante de término de ingeniería en Ambiente y Desarrollo en la Universidad Zamorano, Honduras, desarrolla su trabajo de grado sobre desigualdad social vinculada a la salud ambiental en su país natal, República Dominicana. Trabajó en el Equipo Vargas de Investigación Social y publica artículos de opinión en la revista Afroféminas. 



\section{El mito de la belleza y los cuerpos negros The Myth of Beauty and Black Bodies}

Diana Katherine Moreno Gómez ${ }^{1}$ Isis Yael Amador Campusano ${ }^{2}$

${ }^{1}$ Centro de Estudios Afrodiaspóricos, Universidad Icesi, Colombia

diana.moreno.g@correounivalle.edu.co (https://orcid.org/oooo-0002-4799-8491)

${ }^{2}$ Universidad Zamorano, Honduras

isisyaelamca@gmail.com (https://orcid.org/oooo-0002-0046-242X)

Recibido: 04-04-2021 / Aceptado: 26-05-2021

https://doi.org/10.1880o/conexion.202101.003

\section{PALABRAS CLAVE / KEYWORDS}

Belleza, racismo, mestizaje, negritud, Latinoamérica / Beauty, racism, miscegenation, blackness, Latin America

\section{RESUMEN}

Este artículo presenta reflexiones que permiten analizar el concepto de belleza vinculado a los cuerpos negros y la inclusión o exclusión que estos experimentan a partir del mito de la belleza. Se pretende abordar cómo es percibida la apariencia negra desde las estructuras hegemónicas, cómo se percibe la persona a sí misma y las posibles razones de estas percepciones. Se retoma el mito de la belleza como una construcción política que ha pretendido perpetuar procesos de dominación femenina y cómo, desde el activismo, las comunidades negras han configurado de forma política una contraparte del mito de la belleza que se constituye en proce- sos de reivindicación negra, a través de los cuales es posible reconocer, valorar y dar lugar a la construcción histórica que las mujeres negras hemos realizado sobre el género.

\section{ABSTRACT}

This article presents reflections that allow us to analyze the concept of beauty linked to black bodies and the inclusion or exclusion they experience in this concept from the myth of beauty. It aims to address how the black appearance is perceived from the hegemonic structures, how the person perceives themselves and possible reasons for these perceptions. The myth of beauty is retaken as a political construction that has tried to perpetuate processes of female domination and how, through activism, black communities have politically configured a counterpart of the myth of beauty that is constituted in processes of black vindication, through 
of which it is possible to recognize, value and give rise to the historical construction that black women have made on gender.

\section{El mito de la belleza y los cuerpos negros}

Configurar la experiencia vital desde marcos pensados, estructurados y vividos desde la jerarquización de los miembros de la especie humana en función de su raza, género o clase ha generado a lo largo de la historia procesos de no identificación, a partir de los imaginarios y prejuicios que sustentan una idea de la otredad desde el no-lugar o, como lo denomina Vergara Figueroa (2014), como cuerpos vaciados, que en el encuentro con los discursos hegemónicos son desprovistos de sus saberes y las construcciones que desde allí han tejido para leerse, leer a los otros y leer la vida; son vistos y asumidos desde los prejuicios que se tiene sobre ellos/as y sobre lo que pueden o no hacer. Así pues, el mito de la belleza podría ser entendido como una estrategia que entorpece la edificación de una imagen de sí plena, que permita reconocerse en la singularidad y la diversidad que caracteriza a cada persona, desde la comprensión de los lugares de enunciación, que en términos históricos le han permitido situarse y construir su trayectoria vital.

El mito de la belleza, en tanto constructo social y postulado de género que pretende situar a las mujeres en un mismo lugar, verse, leerse y saberse en posibilidades diversas que escapan a las hegemónicamente pensadas e impuestas para que sean investidas por la mujer. Como lo plantea Davis (1981/2005), el histórico de luchas y oposiciones desplegadas por las mujeres negras en el marco de los procesos de esclavización, posterior a la abolición de esta y hasta la actualidad, dan cuenta de un legado que sustenta un nuevo modelo de feminidad, que dista de características como la pasividad, obediencia, complacencia y debilidad pensadas en torno al ser mujer, ya que estas no reconocen la fuerza, valentía, oposición, dignidad y demás características que permitieron que las mujeres negras se situaran de forma distante frente a las ideologías que históricamente se han impuesto a la mujer.

El interrogante planteado por Sojourner Truth, en 1851, durante la convención por los derechos de las mujeres en Akron, Ohio - ¿Acaso no soy una mujer? (Afribuku, 2018)-, deja entrever las complejidades que supone la definición de género para las mujeres negras, que, con un histórico de deshumanizaciones y tratos «igualitarios» con los hombres en términos del uso de la fuerza y desempeño de labores dentro de la cadena esclavista, no lograban verse en lo que socialmente se definía dentro de la noción mujer.

Su cuestionamiento pone el acento en las contradicciones a partir de las cuales se ha elaborado la construcción de género 
en los cuerpos racializados de las mujeres negras, ya que el modelo hegemónico, que estructura la narrativa sobre lo que es ser mujer, a partir de la idea de feminidad, enuncia «realidades» que distan de la historicidad y genealogía que se han impartido y presentado como referentes a las mujeres negras en el marco de sus cotidianidades.

Si bien las estructuras hegemónicas y sus ideales frente al ser mujer operan en las realidades de las mujeres negras, estas han asumido matices que han implicado la creación de recursos materiales y simbólicos - otros- para construir su ser en medio de tanta desigualdad.

Para acercarse a la comprensión del mito de la belleza en los cuerpos racializados, este artículo sugiere partir de la conceptualización de la ideología del mestizaje como sustento para las elaboraciones que, en torno a la feminidad y el ser mujer, se han estructurado como «verdad» a la cual las mujeres deben acceder, investir y que deben reproducir para ser. Seguidamente, se presenta un breve esbozo del mito de la belleza, en el que se rastrea su procedencia y sus implicaciones en el marco de las contradicciones que sugiere, para sujetas negras racializadas, configurar una identidad de género desde el no-lugar que les otorgan las concepciones sociales sobre lo que es ser mujer. Como tercer apartado, se plantean reflexiones en torno al mito de la belleza, los cuerpos racializados de las mujeres negras y su relación con la construcción subjetiva del ser.

Finalmente, se trazan algunas reflexiones genéricas que pretenden recoger el sentido de la discusión planteada respecto a las posibles formas a partir de las cuales mujeres negras han hecho frente, oposición y resistencia a estas narrativas que pretenden vaciar el histórico de luchas que las sitúan al margen de este mito, con otros elementos por reconocer, visibilizar y honrar como parte de la construcción de género, que sugieren, como lo plantea Davis (1981/2005), una idea otra de feminidad.

Al hablar de mujeres negras enfrentándose, oponiéndose y resistiendo ante el mito de la belleza, es imposible no pensar en Audre Lorde, docente, poeta, feminista y lesbiana afroestadounidense, quien traza el tema de este mito como eje característico transversal en gran parte de su obra. Al respecto, en su poema Quién dijo que era fácil, Lorde (2019) dice:

Tiene tantas raíces el árbol de la rabia que a veces las ramas se quiebran antes de dar frutos.

Sentadas en Nedicks las mujeres se juntan antes de marchar hablan sobre las chicas problemáticas que contratan para ser libres.

Un empleado casi blanco ignora a un hermano que espera para atenderlas primero 
y las damas no se dan cuenta y rechazan los pequeños placeres de su esclavitud.

Pero yo que estoy limitada por mi espejo como por mi cama veo la causa en el color como también en el sexo.

Y me siento acá preguntándome cuál de mis yoes sobrevivirá a todas estas liberaciones.

\section{Género y la ideología del mestizaje}

La ideología del mestizaje se presenta como una política de blanqueamiento en la cual se asume que la población está compuesta por gente blanca, morena, parda, trigueña, negra, entre otros. Esta asunción sugiere que no hay diferencias entre los individuos porque «todos tenemos el negro detrás de la oreja», idea que perpetúa la invisibilización de los grupos negros e indígenas, y fortalece la exclusión y marginalización de estos (Castro de Guerra y Suárez, 2010).

Castellanos-Guerrero (2000) dice sobre este concepto: «La ideología del mestizaje se expresa en discursos que pasan por la negación y la desaparición del indio a través del supuesto mejoramiento biológico de la raza que se pretendía producir con la inmigración europea» (p. 14).

La ideología del mestizaje se ve especialmente reflejada en Latinoamérica y el Cari- de historias de amor romántico entre un hombre blanco y una mujer -no blanca y no rica- como patrón presentado en las telenovelas. Doncel de la Colina y Miranda Villanueva (2017) reconocen la televisión como un elemento de fortalecimiento de esta ideología absurda, ya que son principalmente personas no blancas quienes componen la audiencia de este material audiovisual, lo que afecta sus procesos de identidad y autopercepción, principalmente en jóvenes (Doncel de la Colina y Miranda Villanueva, 2017; Siapera, 2010).

La pareja blanca, siendo «superior», puede ser percibida como una forma de reducción de la opresión. La mujer negra, en el entorno colonial, es socializada principalmente como productora de esclavizados, como objeto para calmar la insurgencia de estos y como madre postiza de las crías blancas de los amos (Albert Batista, 1990/2003). En este contexto, las negras, frente a la pérdida de las crías propias, preferían gestar crías de sus amos blancos; Albert Batista menciona al respecto:

Para la esclava un hijo era una mezcla de dolor y alegría, pues sabía el futuro que les esperaba a sus hijos, ya que muchos, después de los doce o catorce años, eran llevados al mercado para la venta. De ahí que muchas mujeres preferían parir de un blanco o convivir con éste, ya que les aseguraba una mejor suerte a sus hijos y una mejor situación a ellas mismas (1990/2003, p. 14). 
Aunque Allemand (2007) explica que la ideología del mestizaje se inicia en el siglo XIX y se consolida como discurso hegemónico de las Américas luego de las primeras décadas del siglo XX, el mestizo, aun cuando estuviera más cerca de ser blanco, no era blanco; por ende, se da un acercamiento a medias a la cultura, a las ideas, a las prácticas, casi algo tan mítico como la clase media. Como hay una mezcla con una raza «inferior», el grupo se sitúa en un nivel inferior a los referentes blancos.

Según Wade (2013, 2017), el supuesto mestizaje ha provocado que la forma en la que se identifican las colectivas e individuos se vea afectada, aunque estas autopercepciones van de la mano con otros ejes identitarios, como la clase, que tiene un rol ligado a esta ideología e influye en una autopercepción que podría ser engañosa. El autor define el mestizaje como una ideología profundamente racista y jerárquica, ya que se plantea socialmente la blanquitud como punto de referencia ejemplar. Para esta jerarquización, Wade (2013) explica cómo la base de las medidas de valor son blancocéntricas, lo que hace que el desarrollo, el conocimiento, la belleza y lo que valen estos grupos como personas dependan de qué tan cercano a la blanquitud esté el individuo.

El tema del mestizaje recae especialmente sobre el género, ya que está ba- sado en la transmisión de un supuesto intercambio cultural; la base de esta ideología está ligada a la reproducción sexual, la familia, el parentesco y a un «mejoramiento racial», por lo que se hace inevitable que haya una línea que sigue presentando a las personas asignadas femeninas al nacer (o $\mathrm{AFAB}^{1}$ ) como territorios de conquista y vía de mejoramiento, y que refuerza, según Wade, la horizontalización del parentesco, al presentar el proceso de maternidad como totalmente igual al de paternidad. Esta postura es claramente europeizada, concede privilegio de linaje masculino y es patriarcal (Wade, 2013).

Fanon (1952/2009), también hablando de estas líneas que reflejan las jerarquizaciones tanto raciales como de género, menciona la historia popular martiniqueña de una mujer negra sufriendo por el amor imposible que siente hacia un hombre blanco; sobre estas categorías, el autor menciona:

La importancia de estas categorías y el alcance del desdén por los negros y las personas descendientes de negros era, y a menudo es, de tal calibre que, por ejemplo, en la Republica Dominicana, la ley declara que los dominicanos son una población compuesta por blancos e indígenas americanos, todo ello a pesar del genocidio perpetrado por los conquistadores espa- 
ñoles contra los indígenas desde los tiempos de Colón (Fanon, 1952/2009, pp. 231-232).

Sobre la participación política de mujeres indígenas antes de la invasión de los colonos y dejando claro el papel que juega el colonialismo - neo o no- en la disminución de participación política de las mujeres indígenas en los pueblos andinos, Harvey (1989) explica: «Durante el período incaico, las mujeres ocuparon ocasionalmente el cargo de curaca o jefe local, pero los titulares fueron por lo general varones. Después de la conquista, las mujeres se vieron impedidas por completo de ostentar cargos públicos» (pp. 1-2).

El mal llamado Día de la Raza tiene como discurso principal «la celebración del encuentro entre dos culturas» y alude al 12 de octubre, cuando los colonos invadieron territorios donde residían las y los indígenas (Zermeño Padilla, 2008). Según Allemand (2007), el proceso de mestizaje es defendido como algo que apuesta por la civilización y democratización. Entender cómo es presentada esta ideología hace que tenga sentido, entonces, que existan las democracias raciales, que, en el contexto latinoamericano y caribeño, tienen una presencia particularmente fuerte (Walsh, 2010). Esto crea un holograma social de igualdad, ya que evitan a toda costa referirse a la raza, pero prefieren, en su lugar, referirse a la etnicidad y
Por ejemplo, el mito de la democracia racial en Brasil surge como medio de instauración y perpetuación del racismo, a partir de la percepción de que la piel más clara es la ideal; esta línea discriminatoria se ha refugiado en la propaganda de una ausencia de racismo y discriminación. Este mito, según Wade (2013, 2017), se vio reflejado principalmente en el modelo de dominación racial y de género, en el que, otra vez, el hombre blanco escoge mujeres racializadas como medio de «mejoramiento» de la raza.

Respecto a la clasificación del constructo social de lo que es raza, se muestra que estaban los mal llamados indios, los blancos y los mezclados; entre estos, las personas afrodescendientes esclavizadas no contaban, porque no eran consideradas a ese nivel (Walsh, 2010). La raza como tal llega a Latinoamérica y el Caribe con la colonización, por lo que esta distinción entre lo que es superior y lo que es blanco establece el referente, y todos los otros grupos quedan debajo. Esto se asocia a la idea de que, mientras más mezclado está el individuo, más humano y capaz se vuelve.

El mestizaje como ideología representa mejoramiento -blanqueamiento-. Esto se ve incluso en la participación académica, porque la élite económica, intelectual y política de las democracias raciales como Colombia, Venezuela y República Dominicana es blanqueada o blanca. Mientras más blanqueada es, más parti- 
cipa en estos espacios y goza de privilegios en comparación con personas que no fueron socializadas como negras, porque con esta mezcla sus pieles son más claras y sus rasgos son distintos. Esto hace que los niveles de socialización tortuosa que tiene una persona menos favorecida por el sistema causen que las personas negras y no blancas tengan menos presencia al frente de los espacios -académicos o no- denominados antirracistas, mientras que se da más participación a cuerpos blanqueados, que han tenido el privilegio de formarse y de explorar estos espacios.

La ideología del mestizaje se devela y reproduce en las prácticas cotidianas, que tienen cabida dentro de las estructuras en las que participamos. Un ejemplo de este «blanqueamiento» por el que propende el sistema social racializado se hace evidente en la significativa diferencia, que aún persiste en Latinoamérica y el Caribe, en relación con el acceso a la educación superior de la población negra; y, aun cuando se accede a esta, la posibilidad de encontrar narrativas y saber-conocimiento que reconozca la diversidad y aportaciones de las personas negras no es una constante, porque se privilegian los marcos de pensamiento que se han configurado desde y para sostener el racismo estructural.

En la experiencia de una de las autoras de este artículo, en tanto mujer negra colombiana que tuvo el privilegio de acceder a la universidad pública para estudiar psicología, siempre se preguntó por las razones de la ausencia de maestras o maestros negros que pudieran aportar matices a la enseñanza-aprendizaje de este saber, o presentar su trayectoria vital y profesional como referentes en el ejercicio de esta labor; con quienes fuese posible pensar, debatir, construir sobre las implicaciones de acompañar la vida emocional, psíquica o biopsicosocial de las personas negras. Un acompañamiento que, tras un histórico de devaluaciones, luchas, resistencias y resignificaciones, no puede ser genérico.

En esta misma línea, la parlamentaria Ramírez Abella (2007) desarrolló un estudio sobre mujeres afrouruguayas y su liderazgo y participación en el cual presenta hallazgos que dan sentido a muchas asunciones. En primer lugar, de las personas miembros del Poder Ejecutivo y otros organismos del Estado de Uruguay, un $3.5 \%$ eran mujeres afrodescendientes, mientras que la presencia de mujeres no afrodescendientes fue de $5.8 \%$. Por otro lado, la presencia de mujeres afro en la categoría de trabajadores no calificados fue de $41.9 \%$; en contraste, la participación de mujeres no afro en la misma ocupación fue de $24.5 \%$. Por último, en la categoría ocupacional de profesionales, la proporción de afrouruguayas fue de 6.2 $\%$, mientras que la de mujeres no afro fue de $14.1 \%$, aproximadamente ocho puntos porcentuales más. Estos datos dejan entrever una significativa diferencia respec- 
to a las posibilidades de acceso a espacios distintos a los que fueron pensados - por el sistema social racializado- para las personas no blancas.

\section{El mito de la belleza y el lugar de la representación de los cuerpos negros en esta construcción}

De acuerdo con Naomi Wolf (1990/1992), traducida por Cristina Reynoso, el mito de la belleza es una construcción social de la era moderna que se edifica en función de las relaciones de poder que ha establecido el sistema patriarcal para perpetuar estructuras de dominio, pautadas a través de procesos jerárquicos que se materializan en los sistemas de creencias y, con ello, en los prejuicios y estereotipos que las culturas crean y recrean. Según la autora:

La «belleza» es un modelo cambiario, como el patrón oro. Como cualquier economía, está determinada por la política, y en la era moderna occidental es el último y el mejor de los sistemas de creencias que mantienen intacta la dominación masculina. Al asignar valor a las mujeres en una jerarquía vertical de acuerdo a una norma física impuesta culturalmente, se expresan relaciones de poder en las cuales las mujeres deben competir por los recursos que los hombres se han apropiado (1990/1992, p. 217).

Al referirse a este mito, Wolf (1990/1992) a los comportamientos que, en determinado tiempo y espacio, una sociedad considera que son los más apropiados para que una mujer encarne; en el caso de la sociedad occidental, esta idea de belleza está pautada por la ideología dominante. En concordancia con esto, autoras como Manzano García (2012) y Ortiz Piedrahíta (2013) sitúan este dominio al establecer que las estéticas que se privilegian y se encuentran en la cúspide de dicho orden social son las que representan cuerpos esbeltos, cabello rubio y piel blanca; lo racial se ve implicado en la construcción de género, y refuerza las estructuras de poder, al acentuar y replicar la cultura hegemónica y patriarcal blanca.

A efectos de esta reflexión, el mito de la belleza puede ser pensado en tanto ideología a partir de la cual se pretende perpetuar los elementos fundantes de la ideología del mestizaje, dado que este opera bajo la premisa de representaciones sobre lo que puede ser asumido - y no-dentro de la categoría social mujer. Así, el mito de la belleza crea y reproduce intereses y representaciones sociales que apuntan a sostener lo que Davis (1981/2005) ha denominado la matriz de opresiones.

Al revisar las nociones que soportan este mito de la belleza, Manzano García (2012) propone que los ideales de lo bello y lo no bello han pertenecido exclusivamente a la feminidad. Así mismo, el patrón de belleza impuesto por los siglos de discriminación y racismo situó a la belleza 
física como cualidad central a la hora de evaluar a las mujeres, como si estas estuvieran destinadas a continuar siendo, a perpetuidad, referente de belleza por encima de cualquier otra cualidad que las humaniza.

Muñiz (2014) manifiesta que el constructo de feminidad sobre el que se sustentan los ideales de belleza se constituye en un sistema de sumisión y control de las mujeres, por lo que para esta autora la belleza es:

un conjunto de conceptos, representaciones, discursos y prácticas cuya importancia radica en su capacidad performativa en la materialización de los cuerpos sexuados y en la definición de los géneros. [...] La belleza se constituye entonces, en parte de la normalidad femenina que se impone a los cuerpos de las mujeres a través de prácticas identificatorias gobernadas por esquemas reguladores (2014, p. 422).

Para Santiesteban Mosquera (2017) la belleza y lo que se considera por fuera de ella son construcciones que se experimentan en todos los campos de la existencia misma de la persona. Por ello, aunque podrían situarse, en primer momento, desde la corporeidad, en tanto primer territorio para enmarcar y reconocer la existencia, esta construcción se ve imbricada con otras narrativas que sustentan y sitúan la experiencia.
En el marco del abordaje del mito de la belleza, la interseccionalidad permite revisar y cuestionar las elaboraciones que se han construido sobre el ser mujer, y la incidencia de otras dinámicas sociales en ello; así, se logra evidenciar «las experiencias de las mujeres pobres y racializadas como producto de la intersección dinámica entre el sexo/género, la clase y la raza en contextos de dominación construidos históricamente» (Viveros Vigoya, 2016, p. 8). En este sentido, a partir de la representación -entendida como la producción de sentido, significado y narrativas en torno a actos o personas mediante el uso del lenguaje (Hall, 1997)-, se puede leer el mito de la belleza como una categoría construida política, social, cultural e históricamente.

En ese orden, estas representaciones sobre los cuerpos no blancos llegadas y asumidas en el contexto latinoamericano y caribeño se perpetúan contemporáneamente por medio de la idea de que, si lo negro entrara dentro de lo que es bello en medios de consumo, tales como anuncios publicitarios, telenovelas, cine, entre otros, debe ser blanqueado; el blanqueamiento se vuelve un requisito de movilidad social para las personas racializadas y este proceso es una especie de rechazo a lo negro, es decir, a lo propio. Según Gil Hernández (2010), las figuras negras en los medios de comunicación tienden a reproducir la imagen de lo negro como inferior y, tras consumir el producto publicitado, se muestra mejor presentado, si es 
blanqueado, o como ser «exótico», y son las mujeres negras quienes tienden principalmente a encarnar estos personajes.

En esta misma vía, el acercamiento realizado por Santiesteban Mosquera (2017) a lo que ella ha denominado empíricamente como la idiosincrasia del reinado de belleza - para responder a su interrogante ¿qué significa ser bella en Colombia?deja entrever cómo la realización de este certamen anual de belleza instaura, presenta y reproduce un ideal de belleza al cual aspirar.

Para la autora, esta idiosincrasia ha operado como reproductora de una ideología que da cuenta de las relaciones de poder presentes en la construcción de la nación. Santiesteban Mosquera sostiene: «El reinado, de hecho, cohesionó, actualizó, articuló y emplazó, en una coyuntura histórica particular, la dinámica fundamental de la dominación colonial» (2017, p. 90).

En concordancia con los planteamientos de Gil Hernández (2010), no es gratuito, entonces, que, en relación con la posibilidad de verse representada y coronada en el certamen de belleza en Colombia, una mujer negra racializada prefiere definirse a sí misma como una mujer pluriétnica, tal y como lo retoma Santiesteban Mosquera (2017, p. 97).

La permanencia del orden social colonial, la exclusión histórica y social sobre los cuerpos racializados haya perpetuado imaginarios y estereotipos colectivos en torno a la construcción del cuerpo y la persona que es situada desde allí. En este sentido, la construcción de belleza en el caso de las mujeres negras es racista, racializada, sexualizada y, por ende, en esta participan imaginarios, estereotipos y prejuicios que se establecieron sobre los cuerpos negros desde el viaje transatlántico y el proceso de esclavización y la colonia hasta los tiempos actuales.

Para Manzano García (2012), estos estereotipos y prejuicios generan conflictos $y$ contradicciones que funcionan como aniquiladores de identidades plenas, ya que persiste una identidad racial colonial y derogatoria impuesta, desprovista de su propio mundo histórico y cultural, en la que se enseña a mirarse a sí mismo y al otro con los ojos del dominador.

Como menciona Crespo (2011), los prejuicios y estereotipos, que en su mayoría se relacionan con su sexualidad, sensualidad y erotismo, dan cuenta de los procesos de objetivación sexual y patriarcal producto del sistema esclavista y del racismo estructural, a partir de los cuales los cuerpos de las personas negras adquirieron significados específicos que dejan por fuera el reconocimiento de las experiencias diversas de las mujeres negras. Este ejercicio de vaciado permite que la construcción que se hace sobre la otredad y su mundo surja desde los imaginarios 
que la cultura dominante ha creado, y los prejuicios son el principal recurso para dar lugar a esta imagen difusa, incompleta $y$, por ende, dañina de esa otredad diversa (Vergara Figueroa, 2014).

La incidencia de esto en la posibilidad de mirarse, apreciarse, valorarse y reconocerse de forma plena es incalculable, toda vez que la persona racializada se ve desde la propuesta que sustenta los estándares que la cultura dominante ha realizado en torno a su construcción de género. Se pierde de vista el peso de la historicidad en la construcción de las corporalidades racializadas tras la vivencia de los procesos de esclavización y las formas modernas de esta, a través de las cuales las sociedades pretenden perpetuar el poder y la dominación sobre las otredades.

La construcción de saber-conocimiento que Santiesteban Mosquera (2017) realiza en torno a la idea de belleza - de forma situada, en tanto experiencia singular, que habita cada mujer negra racializada y, por ende, no generalizable- puede llevar a pensar en las formas en que, pese al marco presentado por la idiosincrasia del reinado de la belleza en Colombia, una mujer negra puede, en algún momento de la vida, saberse bella de forma distinta y reconocer en su corporeidad, construida desde las incongruencias que desde temprano le presenta el contexto en relación con su ser, un terreno propicio para definir desde allí las estéticas que desea investir. Al igual que LaCigarra, una de las mujeres negras que narran su historia en el texto de Santiesteban Mosquera, hacer parte del proceso afrodiaspórico permitió que una de las autoras de este texto haya podido verse en otro espejo y re-narrar su identidad.

Así, el mito de la belleza se constituye en otra forma de sujeción a los discursos y las construcciones hegemónicas que pretenden invisibilizar las construcciones históricas que han permitido que mujeres negras, a lo largo de una historia de dolor, opresión y degradación, hayan generado acciones para reconocer y dignificar su valentía, fuerza y valía desde un lugar distinto al no-lugar que les fue otorgado.

«Hacer parte o enunciarnos no (me) es suficiente» (Cariño et al., 2017, p. 516). Para una de las autoras de este artículo, enunciarse no es suficiente:

Por ello, resisto siendo una mujer negra en espacios que no fueron hechos para mí: irrumpiendo. En Dominicana, no teniendo la «buena presencia» que exigen los empleadores, ahora en otro país sin tener tampoco maestras que luzcan como yo; siendo una negra estudiando en el extranjero, a poquitos meses de culminar esta etapa, pero con la intermitencia en el sentir si hago parte de. Aun así estando, existiendo, resistiendo. Mi linaje habiendo escapado de las representaciones, siendo ahora todas a través mío lo que a mí me dé la gana. 
La racialización del mito de la belleza y la construcción de la subjetividad de las mujeres negras

Para Viveros Vigoya (2016), el género se ve imbricado en otras categorías sociales, por lo que, al pensar las relaciones sociales y las construcciones individuales, se debe considerar que estas transcurren de formas consubstancial y coextensiva. En esta medida, la interseccionalidad se erige como un recurso para pensar los tejidos que se entretejen en la construcción subjetiva de las personas. Más allá de pensar este recurso como productor de esencialismos o determinismos en las configuraciones subjetivas y el análisis de experiencias, la interseccionalidad permite reconocer las relaciones que se entretejen en las dinámicas de poder.

Abordar el mito de la belleza y plantear reflexiones sobre la forma en la que este opera en las personas racializadas implica revisar el lugar de la representación que se ha construido sobre estos cuerpos y las formas en las que opera en la vida cotidiana. Como lo han planteado el feminismo decolonial y el feminismo negro, la construcción de género no se ha dado de igual forma para los cuerpos racializados; ahí se han visto implicados procesos de interrelación con otras estructuras sociales como la raza y la clase. Por ello, para autoras como Viveros Vigoya (2002), el género no es una cualidad esencial ni estática, sino que es manifestación histórica, que tiene gran variedad de significados en función de las personas, las culturas y los momentos históricos.

Al respecto, el trabajo investigativo realizado por Santiesteban Mosquera (2017) aporta elementos para pensar las diversas formas en las que - un grupo demujeres racializadas, habitantes de la ciudad de Bogotá, en Colombia, hacen frente al no-lugar que supone el no verse o sentirse recogidas en las elaboraciones que componen el ideal de feminidad y el mito de la belleza. En su texto, y en relación con las cuatro trayectorias vitales que retoma la autora, reconoce al menos cuatro estrategias de resistencia que logran trazar estas mujeres para verse y legitimarse, más allá de y pese a los estereotipos que las ubican de forma determinada.

En este sentido, para la autora, la mascarada, el cimarronaje, la escritura y el mestizaje son recursos que, de manera particular, desde los elementos subjetivos de las trayectorias de estas cuatro mujeres, han permitido ofrecer resistencia y generar adaptación, y realizar resignificaciones sobre sí mismas y su historicidad, de modo que han llegado a recuperar su rostro, ante lo que la autora ha definido como la borradura de este.

Si bien la autora no pretende que los planteamientos sobre las historias personales de las mujeres con las que elabora esta narrativa, en torno a su construcción 
subjetiva en un sistema racializado, se conviertan en un análisis rígido y extrapolable a todas las mujeres racializadas, es preciso y oportuno situar su análisis, ya que, a lo largo de la historia, los cuerpos negros racializados han cimarroneado y usado diversas formas narrativas para resistir, crear, plasmar y compartir el saber-experiencia y, a partir de allí, borrar o resignificar las diversas formas de borradura, devolviéndoles el poder desde la posibilidad que otorga narrar la propia experiencia.

En este sentido, leer el mito de la belleza en función de la construcción subjetiva de los cuerpos racializados implica considerar el histórico de deshumanizaciones, degradaciones y prejuicios que se construyeron en torno a la moralidad y percepción de las mujeres negras, vistas como bien común, paridoras, anómalas en relación con el ideal de mujer, entre otras características que las situaban como no humanas (Davis, 1981/2005). E implica también considerar la posibilidad de trasponer los imaginarios que habitan este mito en la construcción real de los cuerpos y experiencias vitales de las mujeres racializadas.

Manzano García (2012) ha planteado que los estereotipos juegan un papel relevante en el proceso de construcción de una identidad; poseen una dinámica propia $\mathrm{y}$ una independencia relativa respecto a su sostén político, económico y social, en correspondencia con la singularidad del desarrollo de la subjetividad humana, donde se acomodan y se reproducen. Estos son habitualmente utilizados por los sectores dominantes de una sociedad para legitimar los antagonismos y las diferencias que se pretenden priorizar con fines de dominación.

Así, el mito de la belleza podría instaurarse en la vida de algunas mujeres racializadas como una atadura que amenaza la búsqueda y consecución de las libertades, y que ocupa un lugar importante en la construcción que se hacen sobre sí mismas; cercena la posibilidad de verse más allá del espejo que propone el opresor, buscando formas para esconder o borrar los vestigios que deja un histórico de degradación que se manifiesta en los modos de decir y en las formas relacionales que la cultura patriarcal y hegemónica propone de forma constante y diversa en las distintas esferas en las que se desarrolla la vida.

\section{Conclusiones: $y$, si no es este el mito de la belleza, ¿cuál sería?}

El cuerpo negro, el cuerpo no blanco, es escenario político, insurgente y de reivindicación frente a la noción occidentalizada y blancocéntrica de lo que es bello y de esta belleza como indicador de cuánto valor tiene este ser - ¿o noser?-. La existencia de este cuerpo, de este «amasijo de cuerdas y tendones» (Rodríguez, 1982), representa el contrapoder, la horizontalización del valor que 
merece y, por ende, moviliza ese cuerpo que es decolonial por su mera existencia. Hernández Basante (2019) menciona cómo las corporalidades negras son encasilladas incluso en un binarismo y circunscritas a una relación genitalidad-sexualidad como forma de dominación. La deshumanización de estos seres afecta principalmente a los cuerpos asignados como femeninos al nacer. Sobre ello, Zapata (2010) muestra en su obra, de forma bastante clara, cómo el proceso de trata de personas desde África establece inmediatamente una desapropiación de los cuerpos negros sobre sí mismos, y sobre ello dice en este fragmento de su escrito Changó, el gran putas:

Insaciables mercaderes
Traficantes de la vida
Vendedores de la muerte
Las Blancas Lobas
Mercaderes de los hombres
Violadoras de mujeres (p. 67).

Tomando el fragmento anterior como punto de partida, la reapropiación de estas personas de sus propios cuerpos representa en sí resistencia; en el caso de muchos grupos, identifican sus mezclas, reconociéndose algunos como afroindígenas o dentro de identidades no blanqueadas, pero renegando o rechazando otras identidades que han sido blanqueadas. Distintos movimientos de mujeres negras, grupos antirracistas, feministas periféricos y decoloniales asumen estas lo «bello» y de lo que tiene valor, y denuncian el racismo, el sexismo, la heteronorma y el clasismo (Curiel, 2007) y hasta la mononorma, y recuperan la valorización de los cuerpos no blancos, estableciéndose en la insurgencia.

Una forma de observar la insurgencia de antaño es la poesía. En su texto Motivos de envidia mulata, Carmen Colón Pellot menciona el tema de la belleza atravesado por el factor racial. Aunque su obra ha sido percibida por ser una especie de exaltación de lo blanco como bello, se puede también asumir como una forma de mostrar cómo es percibida su apariencia no-blanca, y hasta cómo se perciben las no-blancas a sí mismas. ¿Y si fue un llamado de atención y no un salto hacia la inferiorización? El fragmento recuperado en «La poética de la esclavitud (silenciada) en la literatura puertorriqueña: Carmen Colón Pellot, Beatriz Berrocal, Yolanda Arroyo Pizarro y Mayra Santos Febres», de Rivera Casellas (2011), reza:

Tengo envidia de ti, nube blanca;

te enamoras en brazos del viento;

te promiscuas sola con los árboles machos de las sierras altas $\mathrm{y}$ te riman por casta y hermosa.

A mí nadie me canta, a mí me esclavizan las normas; las leyes cristianas (pp. 102-103). 
Dado que la construcción de una idea de sí misma más o menos cohesiva, en la que se incluye la construcción étnico-racial y la de género, es un proceso continuo, que se ve permeado por el acceso a experiencias diversas que permiten configurar la trayectoria vital, la belleza y el mito que se crea en torno a esta, es también un proceso flexible que se elabora y reelabora a partir de los referentes que se puedan tener para cuestionar o cimentar otras fuentes de verificación sobre lo que se instruye como una verdad. A lo largo de la historia, las mujeres negras han asumido posturas de lucha, resistencia y reivindicación que les han permitido develar, en sus cotidianidades, oposiciones a lo establecido.

En la actualidad, algunas colectivas y organizaciones de mujeres negras ${ }^{2}$ a lo largo y ancho de Latinoamérica y el Caribe contemplan dentro de sus líneas de acción la generación de procesos de reconocimiento, pautados por la sensibilidad y la certeza de que las juntazas entre mujeres tienen el poder de establecer un nuevo espejo a través del cual verse, uno que devuelva una imagen cercana a las realidades propias de cada mujer racializada, que no se reconoce y entra en tensión con el sistema ante la imposibilidad de poder desarrollar en libertad las estéticas que dan cuenta de su raíz y su historicidad.

Por ello, se considera importante ir mucho más allá de la representación del discurso de la interseccionalidad; ir más allá del enfoque interseccional es reconocerlo solo como una forma de entender las cosas popularizada por el movimiento feminista radical, pero que tiende a universalizar la experiencia de los cuerpos negros, negando diversas formas de existencia e insurgencia. Wade et al. (2008) hablan sobre cómo es difícil «interrelacionar y hacer evidentes las interrelaciones no sólo teóricas, sino políticas, de la raza y el sexo con la sexualidad, la clase, el lugar geográfico y la historia» (p. 33).

A modo de conclusión, es posible pensar que, en el contexto latinoamericano y caribeño, faltan abordajes más sustanciales de las articulaciones entre raza, género y clase desde el reconocimiento de la experiencia como saber válido para la construcción de conocimiento académico. Entender que las experiencias cotidianas de las mujeres racializadas nacen de las

\footnotetext{
${ }^{2}$ Acción Afro-Dominicana, República Dominicana; AFRITUDE Laboratorio Creativo Político, República Dominicana; Asociación Casa Cultural del Chontaduro, Colombia; Asociación de Mujeres Afrocolombianas (AMAFROCOL), Colombia; Asociación de Mujeres Afrodescendientes de la Vereda Yolombo (ASOMUAFROYO), Colombia; Asociación de Mujeres Afrodescendientes del Norte del Cauca (ASOM), Colombia; Asociación Lila Mujer, Colombia; Asociación de Mujeres Afrocolombianas (AMUAFROC), Colombia; Confederación de Mujeres Campesinas (CONAMUCA), República Dominicana; Grupo Latinoamericano de Estudios, Formación y Acción Feminista (GLEFAS), Latinoamérica y el Caribe; Junta de Prietas, República Dominicana; Kalalú Danza, República Dominicana; Movimiento de Mujeres Dominico-Haitianas (MUDHA), República Dominicana; y el Movimiento de Mujeres Negras «IDENTIDAD» (luego llamado Casa por la Identidad de las Mujeres Afro), República Dominicana.
} 
tensiones, contradicciones, confluencias y estrategias para hacer frente a las demandas que se les imponen desde la lógica del poder permite ampliar el espectro para elaborar una idea de sí que incluya la belleza como un aspecto más en la construcción subjetiva de ser desde una lógica antirracista, feminista y decolonial. 


\section{REFERENCIAS}

Afribuku. (2018, 20 de septiembre). El discurso fundador del feminismo negro: «¿Acaso no soy una mujer?» de Sojourner Truth (Trad. A. de los Santos Pérez). http://www.afribuku.com/ feminismo-negro-estados-unidos-sojourner-truth-acaso-esclavitud/

Albert Batista, C. (2003). Mujer y esclavitud en Santo Domingo (3. ${ }^{\mathrm{a}}$ ed.). Ediciones INDAASEL. (Trabajo original publicado en 1990)

Allemand, P. D. (2007). Quimeras, contradicciones y ambigüedades en la ideología criolla del mestizaje: el caso de José María Samper. Historia y Sociedad, (13), 45-63. https://revistas.unal. edu.co/index.php/hisysoc/article/ view/20437

Cariño, C., Cumes, A., Curiel, O., Garzón, M. T., Mendoza, B., Ochoa, K. y Londoño, A. (2017). Pensar, sentir y hacer pedagogías feministas descoloniales. Diálogos y puntadas. En C. Walsh (Ed.), Pedagogías decoloniales. Prácticas insurgentes de resistir, (re)existir y (re) vivir (tomo 2, pp. 509-536). Ediciones Abya-Yala. http://8.242.217.84:8080/ x m lui/bits tream/hand le/123456789/32966/149.\%20Pedagog\%c3\%adas\%2odecoloniales.pd$\underline{\text { f?sequence }=1 \text { \&isAllowed }=\mathrm{y}}$

Castellanos Guerrero, A. (2000). Antropología y racismo en México. Desacatos, (4), 53-79. http://www.scielo.org.mx/ pdf/desacatos/n4/n4a5.pdf

Castro de Guerra, D. y Suárez, M. M. (2010). Sobre el proceso de mestizaje en Venezuela. Interciencia, 35(9), 654-658. https://www.redalyc.org/ $\mathrm{pdf} / 339 / 33914212004 . \mathrm{pdf}$
Crespo, K. E. (2011). Feminismos y raza, perspectivas desde Puerto Rico. Taller Multiculturalismo y Feminismos, CLADEM.

Curiel, O. (2007). Crítica poscolonial desde las prácticas políticas del feminismo antirracista. Nómadas, (26), 92-101. http://nomadas.ucentral.edu.co/nomadas/pdf/nomadas 26/26 9C Criticaposcolonialdesdelaspracticas.pdf

Davis, A. Y. (2005). Mujeres, raza y clase (Trad. A. Varela Mateos; $2 .^{\mathrm{a}}$ ed.). Ediciones Akal. (Trabajo original publicado en 1981)

Doncel de la Colina, J. A. y Miranda Villanueva, O. M. (2017). Percepción y consumo de telenovelas e identidad étnica de universitarios y universitarias indígenas: el caso de Un refugio para el amor. Comunicación y Sociedad, (30), 265-285. https://doi. org/10.32870/cys.voi3o.6506

Fanon, F. (2009). Piel negra, máscaras blancas (Trads. I. Álvarez Moreno, P. Monleón Alonso y A. Useros Martín). Ediciones Akal. https://lhblog.nuevaradio. org/b2-img/fanon_piel_negra.pdf (Trabajo original publicado en 1952)

Gil Hernández, F. (2010). El «éxito negro» y la «belleza negra» en las páginas sociales. La Manzana de la Discordia, 5(2), 25-44. http://hdl.handle. net $/ 10893 / 3480$

Hall, S. (1997). El trabajo de la representación (Trad. E. Sevilla Casas). En S. Hall (Ed.), Representation: Cultural representations and signifying practices (pp. 13-74). Sage Publications. http:// metamentaldoc.com/14 El trabajo de la representacion_Stuart_Hall. pdf 
Harvey, P. (1989). Género, autoridad y competencia lingüística. Participación política de la mujer en pueblos andinos. Instituto de Estudios Peruanos. http://repositorio.iep.org.pe/handle/ $\underline{\text { IEP/835 }}$

Hernández Basante, K. (2019). Cuerpos insurgentes: territorios de re-existencia de las y los afrodescendientes. $\mathrm{La}$ Manzana de la Discordia, 14(1), 21-35. https://doi.org/10.25100/lamanzanadeladiscordia.v14i1.8297

Lorde, A. (2019). Quién dijo que era fácil. Zindo \& Gafuri. https://www.zindo-gafuri.com/project/quien-dijo-que-erafacil/

Manzano García, M. (2012). Identidad racial: un problema social en nuestros días. Límite. Revista Interdisciplinaria de Filosofía y Psicología, 7(26), 107-119. https://www.redalyc.org/articulo. oa?id=83625847007

Muñiz, E. (2014). Pensar el cuerpo de las mujeres: cuerpo, belleza y feminidad. Una necesaria mirada feminista. Sociedade e Estado, 29(2), 415-432. https://doi.org/10.1590/So102$\underline{69922014000200006}$

Ortiz Piedrahíta, V. (2013). Percepciones y prácticas corporales estéticas de un grupo de jóvenes universitarias afrodescendientes de Cali. Revista CS, (12), 85-125. https://doi.org/10.18046/ recs.i12.1678

Ramírez Abella, B. (2007). Liderazgo y participación política de las mujeres afrodescendientes. http://www.parlamericas.org/uploads/documents/ GATHERING\%205\%20-\%20Ponencia $\% 20-\% 20$ Beatriz $\% 20$ Ramirez $\% 20$ -\%20SPA.pdf
Rivera Casellas, Z. (2011). La poética de la esclavitud (silenciada) en la literatura puertorriqueña: Carmen Colón Pellot, Beatriz Berrocal, Yolanda Arroyo Pizarro y Mayra Santos Febres. Cincinnati Romance Review, 30, 99-116.

Rodríguez, S. (1982). La maza [Canción]. En Unicornio. EGREM.

Santiesteban Mosquera, N. (2017). El color del espejo: narrativas de vida de mujeres negras en Bogotá. Editorial Universidad Icesi y Centro de Estudios Afrodiaspóricos (CEAF).

Siapera, E. (2010). Cultural diversity and global media: The mediation of difference. John Wiley \& Sons.

Vergara Figueroa, A. (2014). Cuerpos y territorios vaciados. ¿En qué consiste el paradigma de la diferencia? ¿Cómo pensamos la diferencia? Revista CS, (13), 338-360. https://doi.org/10.18046/ recs.i13.1830

Viveros Vigoya, M. (2002). De quebradores y cumplidores: sobre hombres, masculinidades y relaciones de género en Colombia. Universidad Nacional de Colombia.

Viveros Vigoya, M. (2016). La interseccionalidad: una aproximación situada a la dominación. Debate Feminista, 52, 1-17. https://doi.org/10.1016/j. df.2016.09.005

Wade, P., Urrea Giraldo, F. y Viveros Vigoya, M. (2008). Identidades racializadas y sexualizadas en América Latina. A manera de introducción. En P. Wade, F. Urrea Giraldo y M. Viveros Vigoya (Eds.), Raza, etnicidad y sexualidades. Ciudadanía y multiculturalismo en América Latina (pp. 17-39). Universidad Nacional de Colombia. 
Wade, P. (2013). Racismo, democracia racial, mestizaje y relaciones de sexo/género. Tabula Rasa, (18), 45-74. https:// doi.org/10.25058/20112742.138

Wade, P. (2017). Estudios afrodescendientes en Latinoamérica: racismo y mestizaje. Tabula Rasa, (27), 23-44. https://doi. org/10.25058/20112742.443

Walsh, C. (2010). Raza, mestizaje y poder: horizontes coloniales pasados y presentes. Crítica y Emancipación, (3), 95-124. http://biblioteca.clacso. edu.ar/ojs/index.php/critica/article/ view/169

Wolf, N. (1992). El mito de la belleza (Trad. C. Reynoso). Debate Feminista, 5. 209-219. http://www.jstor.org/stable/42624047 (Trabajo original publicado en 1990)

Zapata, M. (2010). Changó, el gran putas. Ministerio de Cultura de Colombia.

Zermeño Padilla, G. (2008). Del mestizo al mestizaje: arqueología de un concepto. Memoria y Sociedad, 12(24), 79-95. https://revistas.javeriana.edu. co/index.php/memoysociedad/article/view/8170 